\title{
Characterization of Oxygenated Heterocyclic Compounds and in vitro Antioxidant Activity of Pomelo Essential Oil
}

This article was published in the following Dove Press journal: Drug Design, Development and Therapy

\author{
Guijie Li $\mathbb{D}^{1, *}$ \\ Yujiao Cheng ${ }^{1, *}$ \\ Tenghui Zhang ${ }^{2}$ \\ Yingzhuo $\mathrm{Li}^{3}$ \\ Leng Han' \\ Guolu Liang ${ }^{4}$ \\ 'Citrus Research Institute, Southwest \\ University, Chongqing, People's Republic \\ of China; ${ }^{2}$ Chengdu Centre Testing \\ International Group Co., Ltd., Chengdu, \\ People's Republic of China; ${ }^{3}$ Chongqing \\ Beibei Agricultural and Rural Committee, \\ Chongqing, People's Republic of China; \\ ${ }^{4}$ College of Horticulture and Landscape \\ Architecture, Southwest University, \\ Chongqing, People's Republic of China
}

*These authors contributed equally to this work
Correspondence: Leng Han

Citrus Research Institute, Southwest

University, No. 15 Ganjucun, Xiema,

Beibei District, Chongqing, 4007/2,

People's Republic of China

Email hanleng5@cric.cn

Guolu Liang

College of Horticulture and Landscape

Architecture, Southwest University, No. 2

Tiansheng Road, Beibei District,

Chongqing, 4007I5, People's Republic of

China

Email liangg|@swu.edu.cn
Purpose: Citrus essential oils are widely used for aromatherapy and the alternative treatment of chronic diseases. Beyond the aroma substances, they are known to contain bioactive nonvolatile components; however, little knowledge has been gained about nonvolatiles in the essential oil of pomelo (Citrus grandis Osbeck), the largest citrus fruit. The purpose of this study was to analyze the nonvolatile oxygenated heterocyclic compounds ( $\mathrm{OHCs})$ of pomelo essential oils and evaluate their in vitro antioxidant activities for further development.

Methods: Cold-pressed essential oil (CPEO) and distilled essential oil (DEO) were obtained from the peel of the Liangping pomelo cultivar. High-performance liquid chromatography (HPLC) coupled with a photodiode array and fluorescence detection method was developed to identify and quantify the OHCs of the two essential oils. Ferric reducing antioxidant power and 2,2-diphenyl-1-picrylhydrazyl (DPPH) and 2-phenyl-4,4,5,5-tetramethylimidazoline-1-oxyl 3-oxide (PTIO) radical scavenging assays were used to determine the antioxidative capabilities.

Results: Thirteen OHCs were identified in CPEO. Coumarins such as meranzin $\left(2.0 \mathrm{mmol} \mathrm{L}^{-1}\right)$ and furanocoumarins such as isoimperatorin $\left(1.3 \mathrm{mmol} \mathrm{L}^{-1}\right)$ composed the majority of nonvolatiles in CPEO. These OHCs were characterized by high proportion (58\%) of side chain epoxides. Five OHCs, namely, auraptenol, 6' $7^{\prime}$-dihydroxybergamottin (6', $7^{\prime}$-DHB), imperatorin, isoimperatorin and 8-geranyloxypsoralen were first identified in pomelo CPEO. Eight OHCs were detected at trace amounts in pomelo DEO. Antioxidant assays showed that CPEO was multiple times more potent than DEO regarding the total reducing power and radical scavenging capacity. Clearance of PTIO, a stable reactive oxygen species, followed slow kinetics.

Conclusion: Coumarins and furanocoumarins, two families of $\mathrm{OHCs}$, constituted most of the nonvolatile components in CPEO. The nonvolatiles contributed significantly to the in vitro antioxidant activity of CPEO. Pomelo CPEO showed good prospects as a potential long-lasting natural antioxidant.

Keywords: volatile oil, cold expression, hydrodistillation, psoralen, epoxides, fluorescence, radical scavenging, pummelo, Citrus maxima

\section{Introduction}

Pomelo (Citrus grandis Osbeck), one of the three primordial citrus species, is consumed worldwide and used as a traditional medicine in China and Southeast Asia. Pomelo essential oil has a characteristic odor, and it has numerous applications in fragrant, aromatherapeutic, spiritual and cosmetic fields. ${ }^{1}$ Pomelo essential oil is also suggested to have a broad spectrum of pharmacological activities such as antioxidation, 
inhibition of microbes, ${ }^{2-4}$ antimelanogenesis, ${ }^{5}$ and be used in as an alternative therapy for cough, ulcer, swelling, and epilepsy. ${ }^{6}$

Cold-pressing and water distillation are commonly used methods for the extraction of essential oil from citrus peel. Cold-pressed essential oil (CPEO), a natural mixture of nonvolatile and volatile components, maintains the original composition and properties of phytochemicals. ${ }^{7}$ Distilled essential oil (DEO) lacks the nonvolatile fraction, and its volatile composition varies as a consequence of exposure to different temperatures and acidities during distillation. $^{8}$

Volatiles in both kinds of pomelo essential oils have been intensively studied, and they are mainly composed of mono- and sesquiterpene hydrocarbons and their oxygenated derivatives as well as linear hydrocarbons, alcohols, aldehydes and esters. $^{2,9-11}$ However, only two studies have analyzed a few nonvolatile components of pomelo CPEO. ${ }^{12,13}$ It is known that the nonvolatiles in CPEO of other citrus species such as orange, mandarin, grapefruit, lemon and bergamot, are mainly composed of polymethoxyflavones, furanocoumarins and coumarins. ${ }^{14}$ These three families of compounds all possess a benzo oxygen-containing ring and are collectively called oxygenated heterocyclic compounds (OHCs). An important role in the characterization of citrus essential oils has been attributed to these components. ${ }^{15}$

Reactive oxygen and nitrogen species (ROS and RNS) could damage biomacromolecules such as lipids, proteins and nucleic acids. They are responsible for the degradation of collagen and elastin in dermal tissues. ${ }^{16}$ They also play a pivotal role in the development of age-dependent diseases including atherosclerosis, diabetes, rheumatoid, neurodegenerative disorders and cancers. ${ }^{17}$ To prevent the damage that results from excessive oxygen and nitrogen radicals, the potential of essential oils as antioxidants has been extensively studied and the results have been systematically reviewed; ${ }^{18-20}$ however, limited and inconsistent knowledge has been generated about the role of nonvolatile components in the radical scavenging activities of pomelo essential oil. .,21 $^{2,1}$

Liangping pomelo is one of the representative cultivars of Chinese pomelos. ${ }^{22}$ This study aimed to provide preliminary evidence for the potential antioxidant therapy using pomelo essential oil and/or its major bioactive components. The first objective was to characterize the profile of nonvolatile OHCs in cold-pressed and distilled essential oils of Liangping pomelo. The second objective was to evaluate the in vitro antioxidant activities of the essential oils and determine the contribution of nonvolatiles to the activity of CPEO.

\section{Materials and Methods}

\section{Pomelo Samples and Preparation of Essential Oil}

Fresh mature fruits of Liangping pomelo were obtained from an orchard of the National Citrus Germplasm Repository (106 $43^{\prime}$ E, 29 $83^{\prime} \mathrm{N}$; altitude $241 \mathrm{~m}$ ). Coldpressed essential oil (CPEO) was obtained by using a method as described in our previous study. ${ }^{23}$ Briefly, the fruit flavedo layer was manually peeled and extracted using a household squeezer. The aqueous peel oil emulsion was collected and quickly centrifuged at $12,000 \mathrm{rpm}$ at $4^{\circ}$ $\mathrm{C}$ for $10 \mathrm{~min}$. The upper-most oil layer was then collected. Distilled essential oil (DEO) was obtained by using an improved Clevenger apparatus with a second condenser as described by Chen et al. ${ }^{24}$ One hundred grams of pomelo flavedo was distilled with $0.4 \mathrm{~L}$ of water until no more essential oil was obtained. The collected CPEO and DEO were dried using anhydrous sodium sulfate and stored at $-80^{\circ} \mathrm{C}$ for two weeks to remove any sediment.

\section{Chemicals}

HPLC-grade solvents and reagents: acetonitrile, methanol, tetrahydrofuran and n-hexane were purchased from Honeywell (Morris Plains, USA). Dimethyl sulfoxide and phosphoric acid were purchased from Sigma-Aldrich ( $\mathrm{St}$ Louis, USA) and Chron Chemicals (Chengdu, China), respectively. Ultrapure water was freshly produced using a Milli-Q plus Advantage A10 system (Molsheim, France).

Analytical pure reagents, ferric chloride hexahydrate, 2,4,6-tripyridyl-s-triazine (TPTZ), trichloroacetic acid, acetone, and anhydrous ethanol were purchased from Taixin Chemical (Chongqing, China). 2.2-Diphenyl1-picrylhydrazyl (DPPH) and ascorbic acid were purchased from Yuanye Biotech (Shanghai China). 2-Phenyl-4,4,5,5-tetramethyl-imidazoline-1-oxyl 3-oxide (PTIO) was purchased from TCI Chemicals (Shanghai, China).

Standard substances of oxygenated heterocyclic compounds (OHCs): meranzin hydrate, auraptenol, meranzin, isomeranzin, osthole, oxypeucedanin, 3,5,6,7,8,3',4'heptamethoxyflavone, 6',7'-dihydroxybergamottin, isoimperatorin, imperatorin, 8-geranyloxypsoralen, auraptene 
and psoralen were purchased from Yuanye Biotech (Shanghai China). Tangeretin and 6',7'-epoxybergamottin were purchased from ChromaDex (Irvine, USA). Nobiletin was purchased from TRC (Toronto, Canada). $6^{\prime}, 7^{\prime}$-Epoxyauraptene was obtained by using a preparative liquid chromatography separation from pomelo CPEO (details can be found in Supplementary Information. Other related nonvolatile standards such as phenolic acids and flavanone glycosides were purchased from CRMRM, Ltd. (Beijing, China). The stock solution of the individual standard was approximately $1 \mathrm{mg} \mathrm{mL}^{-1}$ in methanol or dimethyl sulfoxide and was stored at $-20^{\circ} \mathrm{C}$.

\section{Analytical HPLC Condition and Settings}

A Thermo Accucore PFP column (i.d. $4.6 \times 150 \mathrm{~mm}, 2.6$ $\mu \mathrm{m})$ attached to an Agilent EC-C18 guard column (i.d. $4.6 \times 5 \mathrm{~mm}, 2.7 \mu \mathrm{m}$ ) was used with an Agilent 1260 liquid chromatography system equipped with a photodiode array (PDA) detector and a fluorescence detector (FLD) in series. The mobile phase consisted of $0.025 \%$ phosphoric acid aqueous solution (A), methanol (B), acetonitrile (C) and a mixture of water/acetonitrile/tetrahydrofuran (volume ratio 55/20/25, D) at $1 \mathrm{~mL} / \mathrm{min}$ flow rate. Column temperature was maintained at $30^{\circ} \mathrm{C}$. The LC program to separate the nonvolatile components of pomelo essential oils consisted of the following linear gradients: 0-7 min, 33\% B and 4\% C; 7-20 min, 33-0\% B, 4-34\% $\mathrm{C}$ and $0-25 \% \mathrm{D} ; 20-25 \mathrm{~min}, 34-40 \% \mathrm{C}$ and $25 \% \mathrm{D}$; 25-30 $\mathrm{min}, 40-75 \% \mathrm{C}$ and $25-0 \% \mathrm{D} ; 30-35 \mathrm{~min}$, 75-100\% C; and 35-40 $\mathrm{min}, 100 \% \mathrm{C}$; after $40 \mathrm{~min}$, the initial conditions were reestablished. Solvent A was used to make up the unlisted percentage in the gradient. Detectors were set following our previous work with minor modifications. ${ }^{25}$ PDA was set to scan 210-400 nm. FLD was set to excite at $330 \mathrm{~nm}$ and scan the emission wavelengths 340-560 nm. Essential oil samples were diluted in hexane at proper volume ratios prior to HPLC analysis.

\section{Identification and Quantitation of OHCs in Essential Oils}

A combination of spectral and chromatographic retention information was used for the identification of sample peaks. The UV spectrum and fluorescence (FL) emission spectrum of all standard compounds were scanned and composed a homemade library. The identity of a sample peak was preliminarily confirmed by evaluating the matching degree of UV and FL spectra compared to standards using an OpenLab CDS ChemStation software (Agilent, USA). Identification was further confirmed by checking the consistency of retention time.

Quantitation of the sample OHCs was carried out using an internal standard method. Psoralen was added as the internal standard (IS) at a concentration of $0.27 \mathrm{mmol} \mathrm{L}^{-1}$ to each level of the standard calibration solution, and the correction factor (CF) was determined. The same level of IS was added to essential oil samples and OHCs were quantitated using the CFs.

\section{Validation of Analytical Methods}

Validation of the developed analytic procedures was carried out according to the International Conference on Harmonization guidelines. ${ }^{26}$ Linearity, limit of detection (LOD), limit of quantitation (LOQ), and spiking recovery were determined.

\section{Ferric Reducing Antioxidant Power Assay} The FRAP analysis was conducted spectrophotometrically as suggested by Benzie and $\operatorname{Strain}^{27}$ with a few modifications. Briefly, $300 \mathrm{mmol} \mathrm{L}^{-1}$ acetate buffer adjusted to $\mathrm{pH} 3.6,10 \mathrm{mmol} \mathrm{\textrm {L } ^ { - 1 }}$ TPTZ dissolved in 40 mmol L ${ }^{-1} \mathrm{HCl}$ and $20 \mathrm{mmol} \mathrm{L}{ }^{-1} \mathrm{FeCl}_{3} \cdot 6 \mathrm{H}_{2} \mathrm{O}$ were mixed at a ratio of 10:1:1 to form the working solution. The essential oils and the negative control, D-limonene, were diluted in a mixture of acetone and methanol $(50: 50, \mathrm{v} / \mathrm{v})$ to form a series of dilutions at $10 \%(\mathrm{v} / \mathrm{v})$ intervals. The positive control, ascorbic acid, was dissolved in the same mixed solvent at a concentration of $227.1 \mu \mathrm{mol} \mathrm{L}^{-1}$. Fifty microliters of each solution were vortexed vigorously with $950 \mu \mathrm{L}$ of the TPTZ working solution for $6 \mathrm{~min}$ in the dark. The absorbance at $593 \mathrm{~nm}$ was recorded using a Varioskan LUX microplate reader (Thermo Scientific, USA). The FRAP value was determined using ascorbic acid calibration and expressed as micromoles of ascorbate equivalent antioxidant capacity (AEAC) per liter of essential oil.

\section{DPPH Free Radical Assay}

The DPPH assay was carried out following the procedure described by Brand-Williams et $\mathrm{al}^{28}$ with minor modifications. Then, $0.1 \mathrm{~mL}$ of essential oil dilution at different concentrations as described in FRAP analysis was added to $0.7 \mathrm{~mL}$ of $63.4 \mu \mathrm{mol} \mathrm{L}{ }^{-1}$ DPPH methanol solution. The mixture was vortexed vigorously in the dark for $120 \mathrm{~min}$ 
to ensure a thorough reaction as reported by $\mathrm{Du}$ et al. ${ }^{29}$ Absorbances were measured at $517 \mathrm{~nm}$. The scavenging effect was calculated using the following equation:

Scavenging effect $(\%)=\left(1-\frac{A_{\text {reaction mixture }}-A_{\text {sample }}}{A_{\text {blank }}}\right) \times 100$

where $\mathrm{A}_{\text {reaction mixture }}$ is the absorbance of sample and DPPH mixture after the reaction; DPPH solution was replaced with absolute methanol and $\mathrm{A}_{\text {sample }}$ is the absorbance of the sample and methanol mixture; essential oil sample was replaced by the acetone/methanol solvent and A blank was the absorbance of the solvent and DPPH mixture. Ascorbic acid and D-limonene were used as the positive and negative controls, respectively. The amount of antioxidant necessary to decrease the initial DPPH concentration by half, 50\% efficient dose (ED50), was calculated. Scavenging capability was expressed as anti-radical power (ARP), which is equal to $1 /$ ED50.

\section{PTIO Free Radical Assay}

A PTIO radical scavenging assay was conducted following the protocol described by $\mathrm{Li}^{30}$ with modification. PTIO was dissolved in methanol at $214.3 \mu \mathrm{mol} \mathrm{L^{-1 }}$. Exactly $0.2 \mathrm{~mL}$ essential oil dilution at different concentrations was separately added to $0.4 \mathrm{~mL}$ of the PTIO methanol solution, and the mixture was vortexed at $37^{\circ} \mathrm{C}$ for 6 h. Absorbance was measured at $586 \mathrm{~nm}$. The PTIO scavenging effect was calculated using the same equation as for DPPH. Again, ascorbic acid and D-limonene were used as the positive and negative controls, respectively.

\section{Statistical Analysis}

All experiments were performed in triplicate and the results are presented as the mean \pm standard deviation. Differences between the mean values for the individual groups were assessed by a one-way analysis of variance (ANOVA) with Duncan's multiple range tests. $\mathrm{P}<0.05$ was considered as a statistically significant difference. SPSS 22 software (IBM Corporation, USA) was used for the analysis.

\section{Results and Discussion \\ LC Separation of OHCs}

The structural similarity of these oxygenated heterocyclic compounds made it impractical to chromatographically separate all of them under isocratic solvent conditions. Moreover, isomers such as auraptenol, meranzin and isomeranzin eluted at almost the same time in a watermethanol or water-acetonitrile gradient. Therefore, an additional organic phase, tetrahydrofuran, was introduced, and a quaternary solvent gradient was developed.

The optimized separation of OHCs, the internal standard (IS) and a few other nonvolatile components in the CPEO of Liangping pomelo is shown in Figure 1. Both chromatograms of UV absorbance monitored at $325 \mathrm{~nm}$ and fluorescence (FL) emission monitored at $395 \mathrm{~nm}$ are presented. The FL emission response was inverted for a better comparison with the UV response, and large FL peaks were truncated so that small peaks were clearly shown. The retention times of FL peaks are slightly later than the corresponding UV peaks because of the series connection of PDA and FLD. As seen in the figure, from meranzin hydrate to auraptene, sixteen $\mathrm{OHCs}$, including three polymethoxyflavones, ie nobiletin, heptamethoxyflavone and tangeretin which were not detected in the essential oil sample, were chromatographically resolved.

\section{Identification of Sample OHCs}

To identify sample OHCs in the presence of other nonvolatiles using less selective detectors such as PDA and FLD, it is necessary to check their full spectra of both UV absorbance and FL emission and compare them to those of standards. ${ }^{25} \mathrm{UV}$ and FL full spectra of the sixteen $\mathrm{OHC}$ standards were collected and are shown in Figure 2. All Spectra are categorized in five groups (A-E). Compounds within a group have similar spectra and substitution patterns. For instance, the five 7,8-disubstituted coumarins all have UV maxima at 322 325 $\mathrm{nm}$ and a small shoulder peak at $245 \sim 255 \mathrm{~nm}$, and their FL maxima are approximately 394 400 nm (Figure 2A). By checking either the UV or FL spectrum of a sample peak, it might not be able to make a correct identification from the five candidates; however, when the two spectra are used together as a double insurance, the possibility of erroneous identification will be minimized. In addition, the chromatographic retention time was always another independent measurement to confirm identification.

Pomelo peel has been shown to contain flavanones, flavones, flavanols, coumarins and furanocoumarins, in both the free and glycosidic forms. ${ }^{31,32}$ However, current knowledge of pomelo oil nonvolatiles was limited. By using the above described tools together, we identified six furanocoumarins namely oxypeucedanin, $6^{\prime}, 7^{\prime}-$ dihydroxybergamottin $\left(6^{\prime}, 7^{\prime}\right.$-DHB), imperatorin, isoimperatorin, epoxybergamottin and 8-geranyloxypsoralen (8-GOP), and seven coumarins namely meranzin hydrate, auraptenol, meranzin, isomeranzin, 6', $7^{\prime}-$ 


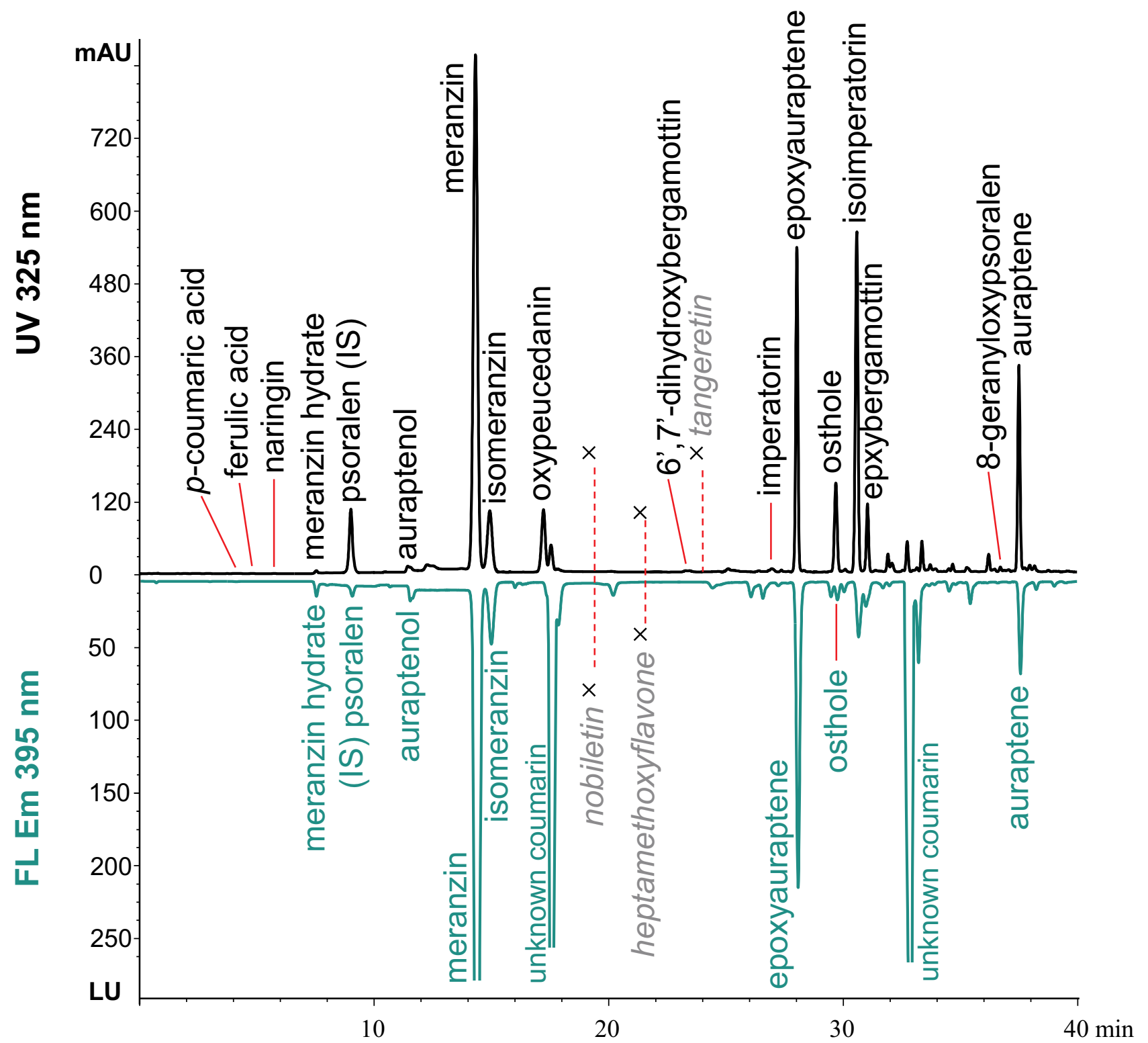

Figure I Separation and identification of nonvolatiles in the cold-pressed essential oil of Liangping pomelo. Chromatograms of UV absorbance monitored at 325 nm and fluorescence emission (FL Em) monitored at $395 \mathrm{~nm}$ are shown in reverse. Nobiletin, heptamethoxyflavone, and tangeretin are not found and marked with $\times$.

epoxyauraptene, osthole and auraptene in the CPEO sample (Figure 1). Compared with previous studies, ${ }^{12,13}$ this is the first report of auraptenol, $6^{\prime}, 7^{\prime}$ DHB, isoimperatorin, imperatorin and 8-GOP in pomelo CPEO. In addition, two peaks at retention times of 17.6 min and 32.9 min were tentatively identified as coumarins according to their UV and FL spectra. Therefore, the nonvolatile constituents in Liangping pomelo CPEO were mainly composed of furanocoumarins and coumarins. Two phenolic acids (p-coumaric and ferulic acids) and a flavanone glycoside (naringin) were detected in trace amounts.

\section{Quantitative Analysis of Coumarins and} Furanocoumarins

Quantitation wavelengths of the OHCs were set at or near their spectral maxima (Figure 2). The linear range, CF, LOD and LOQ of UV and fluorescence responses of each standard compound were determined and are shown in Table 1. Except for tangeretin which does not fluoresce, all the other OHCs showed commensurate results (relative error $<5 \%$ ) when quantitated using UV and FL signals within the overlapping linear ranges.

The total amount and molar concentrations of each coumarin and furanocoumarin in cold-pressed and 

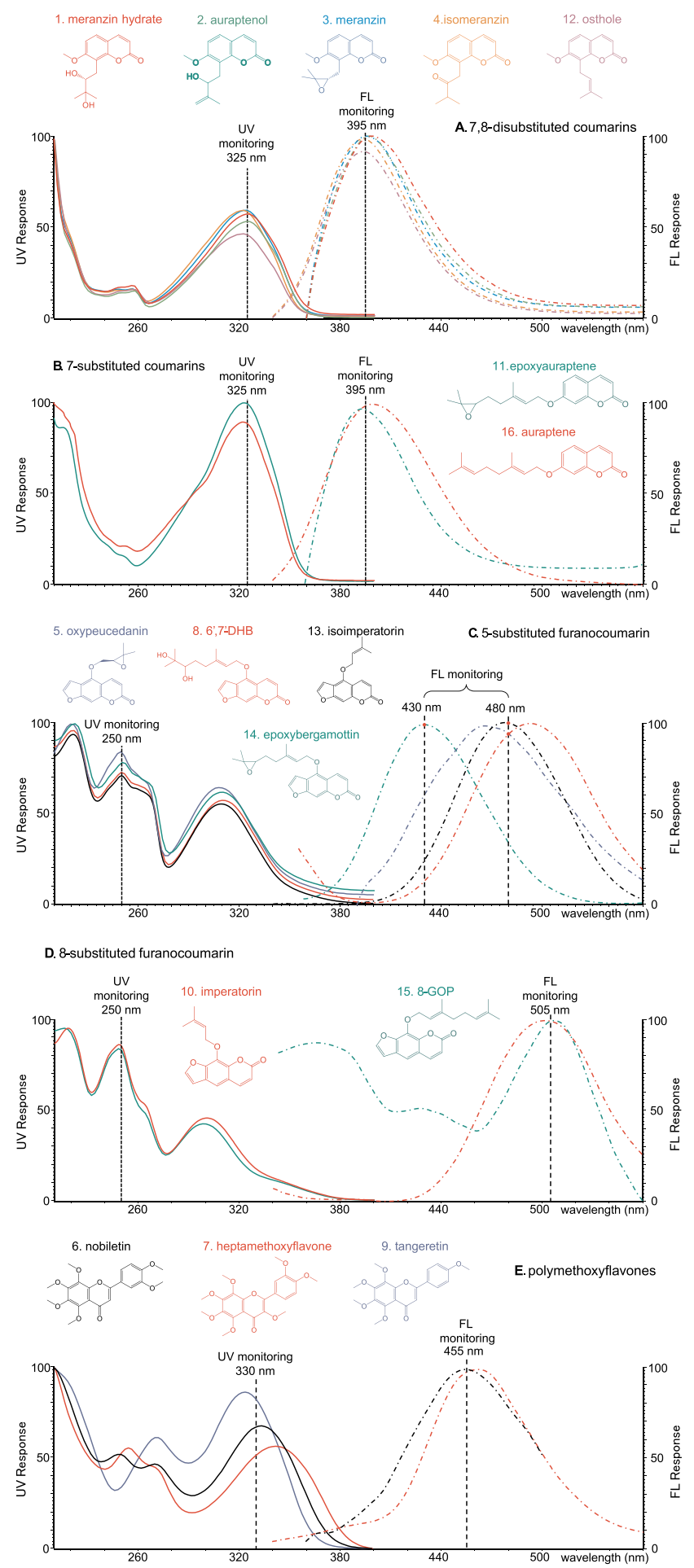

Figure 2 UV absorbance and fluorescence emission spectra of 16 oxygenated heterocyclic compound standards categorized into five groups according to their molecular structure. (A) 7.8-disubstituted coumarins; (B) 7-substituted coumarins; (C) 5-substituted furanocoumarins; (D) 8-substituted furanocoumarins; (E) polymethoxyflavones.

distilled essential oils of Liangping pomelo are shown in Table 1. To the best of our knowledge, this table shows the most comprehensive qualitative and quantitative information of the major oxygenated heterocyclic components in pomelo essential oils. The most abundant compounds in CPEO are meranzin and isoimperatorin $\left(2.0 \mathrm{mmol} \mathrm{L}^{-1}\right.$ and $\left.1.3 \mathrm{mmol} \mathrm{L}^{-1}\right)$, followed by $6^{\prime}, 7^{\prime}$-epoxyauraptene $\left(0.62 \mathrm{mmol} \mathrm{L}^{-1}\right)$ and auraptene $\left(0.51 \mathrm{mmol} \mathrm{L}^{-1}\right)$. Only seven $\mathrm{OHCs}$ were detected at very low concentrations in DEO. The accuracy of quantitation was verified by a standard spiking recovery test, and the results are shown in Supplementary Table S1. Recovery rates in CPEO ranged between $89.6 \%$ and $105.4 \%$, and those in DEO ranged between $80.7 \%$ and $118.7 \%$.

Pomelo CPEO is characterized by high contents of coumarin and furanocoumarin epoxides. Meranzin, oxypeucedanin, 6',7'-epoxyauraptene and epoxybergamottin collectively accounted for more than half (58\%) of the total OHCs $(\mathrm{mol} / \mathrm{mol})$. The OHC profile in CPEO of other commercially important citrus species was summarized by Dugo and Russo. ${ }^{33}$ Sweet orange (Citrus sinensis) or mandarin ( $C$. reticulata) CPEO contains no epoxides. Bergamot (C. bergamia), lime (C. aurantifolia) and lemon (C. limon) oils contain low proportions of epoxides $(0.3 \% \sim 8.3 \%, 3.3 \% \sim 7.8 \%$ and $7.8 \% \sim 41.8 \%$ respectively). OHCs of bitter orange (C. aurantium) and grapefruit (C. paradisi) CPEOs contain comparable amounts $(29.9 \% \sim 66.5 \%$ and $38.3 \%$ $\sim 64.8 \%$ ) of total epoxides as pomelo oil; however, these two oils lack oxypeucedanin. Therefore, the qualitative and quantitative composition of OHCs can be used as a criterion to discriminate pomelo CPEO from the other citrus oils.

Furanocoumarins appear to be responsible for the majority of phototoxic reactions in humans caused by plants. The phototoxic action of these compounds is due to their ability to react with nucleobases of DNA under UVA irradiation, giving rise to cross-links in DNA strands. ${ }^{34}$ The confirmed phototoxic and photomutagenic compounds include bergapten (5-methoxypsoralen, 5-MOP), psoralen and xanthotoxin (8-methoxypsoralen). ${ }^{35}$ In the current study we did not find these compounds in the CPEO or DEO of the pomelo. Other potential congeners, ie isoimperatorin, oxypeucedanin and epoxybergamottin, whose contents were approximately 350, 110 and $85 \mathrm{mg} / \mathrm{L} \mathrm{(ppm)} \mathrm{respectively,} \mathrm{were} \mathrm{the} \mathrm{most} \mathrm{abundant} \mathrm{fur-}$ anocoumarins in the CPEO sample. However, isoimperatorin was reported to be phototoxic to rabbits at a threshold of $5000 \mathrm{ppm}$, and oxypeucedanin had a threshold of 250 ppm to Guinea pigs and rabbits. ${ }^{36}$ A recent cohort study 
Table I Linear Range, Correction Factor, Limit of Detection and Quantitation of $16 \mathrm{OHC}$ Standards and Their Concentrations in the Pomelo Essential Oils

\begin{tabular}{|c|c|c|c|c|c|c|c|c|c|c|c|}
\hline \multirow[t]{2}{*}{ No. } & \multirow[t]{2}{*}{ Compound Name } & \multicolumn{4}{|c|}{ UV Detection } & \multicolumn{4}{|c|}{ FL Detection } & \multicolumn{2}{|c|}{$\begin{array}{c}\text { Average } \\
\text { Concentration }^{\mathrm{a}}\end{array}$} \\
\hline & & $\begin{array}{c}\text { Range } \\
(\mu \mathrm{mol} \\
\left.\mathbf{L}^{-1}\right)\end{array}$ & CF & $\begin{array}{c}\text { LOD } \\
(\mu \mathrm{mol} \\
\left.\mathbf{L}^{-1}\right)\end{array}$ & $\begin{array}{c}\text { LOQ } \\
(\mu \mathrm{mol} \\
\left.\mathbf{L}^{-1}\right)\end{array}$ & $\begin{array}{c}\text { Range } \\
(\mu \mathrm{mol} \\
\left.\mathrm{L}^{-1}\right)\end{array}$ & CF & $\begin{array}{c}\text { LOD } \\
(\mu \mathrm{mol} \\
\left.\mathbf{L}^{-1}\right)\end{array}$ & $\begin{array}{c}\text { LOQ } \\
(\mu \mathrm{mol} \\
\left.\mathbf{L}^{-1}\right)\end{array}$ & $\begin{array}{c}\text { CPEO } \\
(\mu \mathrm{mol} \\
\left.\mathbf{L}^{-1}\right)\end{array}$ & $\begin{array}{c}\text { DEO } \\
(\mu \mathrm{mol} \\
\left.\mathbf{L}^{-1}\right)\end{array}$ \\
\hline 1 & Meranzin hydrate & $1.8-1800$ & 8.07 & 0.57 & 1.98 & $0.1-360$ & 1.600 & 0.03 & 0.09 & $\begin{array}{l}20.4 \\
\pm 3.0\end{array}$ & ND \\
\hline 2 & Auraptenol & $1.9-1100$ & 9.49 & 0.65 & 2.15 & $0.1-380$ & 1.359 & 0.04 & 0.12 & $\begin{array}{l}58.0 \\
\pm 0.5\end{array}$ & ND \\
\hline 3 & Meranzin & $0.8-1900$ & II.44 & 0.15 & 0.46 & $0.8-1920$ & 0.858 & 0.005 & 0.02 & $\begin{array}{c}1995.6 \\
\pm 28.5\end{array}$ & $3.9 \pm 0.3$ \\
\hline 4 & Isomeranzin & $0.2-1900$ & 11.84 & 0.15 & 0.54 & $0.04-190$ & 8.529 & 0.01 & 0.03 & $\begin{array}{c}264.9 \\
\pm 4.1\end{array}$ & $0.5 \pm 0.1$ \\
\hline 5 & Oxypeucedanin & $3.5-1750$ & 37.68 & 0.14 & 0.49 & $17.5-1750$ & 0.101 & 0.45 & 1.50 & $\begin{array}{c}374.7 \\
\pm 5.5\end{array}$ & $\begin{array}{c}0.6 \\
\pm 0.2^{\mathrm{b}}\end{array}$ \\
\hline 6 & Nobiletin & $5.0-1200$ & 21.57 & 0.22 & 0.75 & $12.4-1240$ & 0.142 & 0.37 & 1.27 & ND & ND \\
\hline 7 & Heptamethoxyflavone & $2.3-1160$ & 27.87 & 0.07 & 0.25 & $23.1-1150$ & 0.051 & 0.95 & 3.33 & ND & ND \\
\hline 8 & $6^{\prime}, 7^{\prime}-\mathrm{DHB}$ & $0.3-2700$ & 22.81 & 0.03 & 0.11 & $2.7-1600$ & 0.024 & 0.11 & 0.40 & $\begin{array}{l}11.2 \\
\pm 0.9\end{array}$ & ND \\
\hline 9 & Tangeretin & $1.3-1340$ & 26.40 & 0.05 & 0.16 & NA & NA & NA & NA & ND & ND \\
\hline 10 & Imperatorin & $0.17-740$ & 19.60 & 0.07 & 0.18 & $55.5-5550$ & 0.002 & 17.65 & 58.83 & $\begin{array}{l}27.5 \\
\pm 0.3\end{array}$ & ND \\
\hline II & $6^{\prime}, 7^{\prime}$-epoxyauraptene & $3.2-1600$ & 12.36 & 0.06 & 0.25 & $0.6-1600$ & 0.696 & 0.02 & 0.06 & $\begin{array}{l}622.2 \\
\pm 10.3\end{array}$ & $0.6 \pm 0.1$ \\
\hline 12 & Osthole & $2.1-2050$ & 11.55 & 0.08 & 0.33 & 4.I-2050 & 0.290 & 0.20 & 0.61 & $\begin{array}{c}168.8 \\
\pm 2.8\end{array}$ & $<\mathrm{LOQ}$ \\
\hline 13 & Isoimperatorin & $0.7-1850$ & 30.34 & 0.04 & 0.11 & $5.5-11,100$ & 0.018 & 1.00 & 3.29 & $\begin{array}{l}1289.3 \\
\pm 23.9\end{array}$ & $2.3 \pm 0.1$ \\
\hline 14 & Epoxybergamottin & $0.3-1400$ & 27.98 & 0.11 & 0.37 & $5.6-1410$ & 0.095 & 0.31 & 1.07 & $\begin{array}{l}240.7 \\
\pm 4.5\end{array}$ & $\begin{array}{c}0.4 \\
\pm 0.0^{\mathrm{b}}\end{array}$ \\
\hline 15 & 8-GOP & $0.6-600$ & 18.54 & 0.03 & 0.12 & $59.1-1500$ & 0.001 & 30.14 & 70.92 & $7.9 \pm 0.2$ & ND \\
\hline 16 & Auraptene & $3.4-1700$ & 7.28 & 0.10 & 0.37 & $0.7-1680$ & 0.481 & 0.03 & 0.10 & $\begin{array}{l}513.5 \\
\pm 10.3\end{array}$ & $0.4 \pm 0.1$ \\
\hline & Total & & & & & & & & & $\begin{array}{l}\mathrm{I} .56 \\
g L^{-1}\end{array}$ & $\begin{array}{c}2.4 \mathrm{mg} \\
\mathrm{L}^{-1}\end{array}$ \\
\hline
\end{tabular}

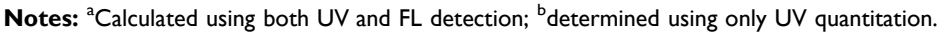

Abbreviations: FL, fluorescence; ND, not detected; DHB, dihydroxybergamottin; NA, not available; GOP, geranyloxypsoralen.

found no significant association between the risks of melanoma or squamous cell carcinoma and dietary intake of furanocoumarins (including epoxybergamottin). ${ }^{37}$ Although there is still not enough information to identify human safety thresholds of essential oils that contain various furanocoumarins, current data suggest that safety issues should not be of major concern when pomelo essential oils are topically applied. 
Table 2 Antioxidant Capacities of Liangping Pomelo Essential Oils Obtained from Cold Expression and Distillation

\begin{tabular}{|c|c|c|c|}
\hline Samples & 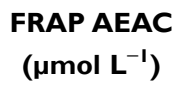 & 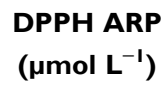 & $\begin{array}{l}\text { PTIO ARP } \\
\left(\mu \mathrm{mol} \mathrm{L^{-1 }}\right)\end{array}$ \\
\hline CPEO & $459.9 \pm 10.0^{a}$ & $611.9 \pm 19.4^{a}$ & $862.8 \pm 14.6^{a}$ \\
\hline DEO & $102.2 \pm 5.4^{\mathrm{b}}$ & $265.1 \pm 17.1^{b}$ & $335.5 \pm 6.0^{b}$ \\
\hline D-limonene & $29.8 \pm 0.3^{c}$ & ND & ND \\
\hline
\end{tabular}

Note: ${ }^{\mathrm{a}, \mathrm{b}, \mathrm{c}}$ Different letters in the same column indicate significant differences $(P<$ 0.05).

Abbreviations: AEAC, ascorbic equivalent antioxidant capacity; ARP, anti-radical power, the equivalent amount of ascorbic acid to remove $50 \%$ of free radicals; ND, not determined under the current experimental conditions.

\section{Reducing Power and DPPH and PTIO Scavenging Activity of Essential Oils}

The total reducing power of oil samples expressed as ascorbic equivalents is shown in Table 2 . The reducing power of CPEO was approximately 4.5 times higher than that of DEO and 15 times higher than that of D-limonene, which is the prevailing (normally $>90 \%$ ) volatile component of citrus essential oils.

The results of the DPPH scavenging effect as a function of sample amount are shown in Figure 3. The top scales indicate the consumed sample volume per $\mu \mathrm{mol}$ of DPPH; the bottom scales indicate the molar ratios of ascorbic acid to DPPH. As seen in the figure, D-limonene exhibited weak scavenging activity and could not reach a $50 \%$ clearance of DPPH. An early study of citrus

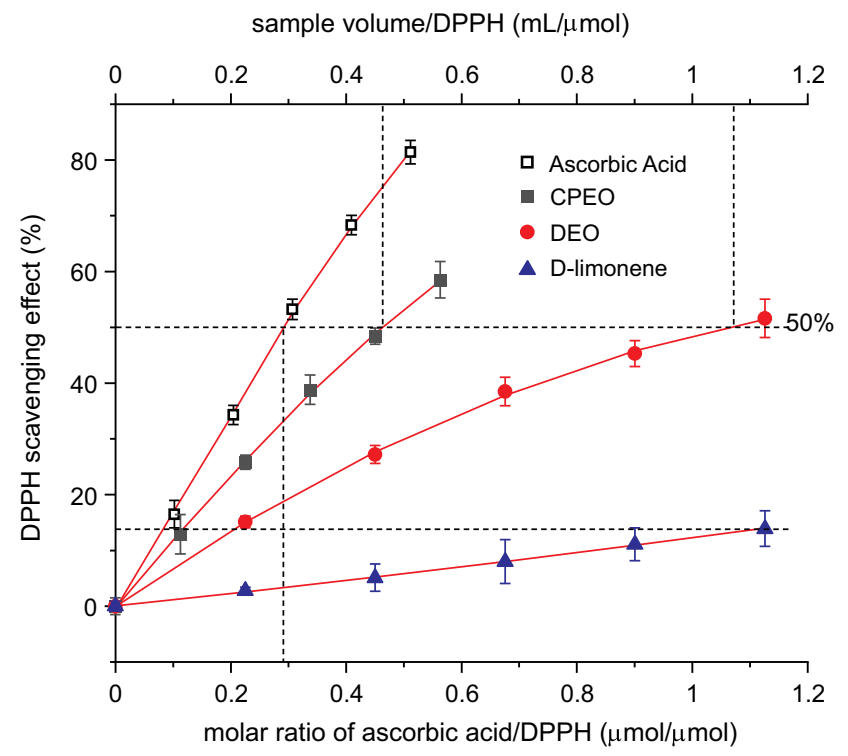

Figure 3 DPPH scavenging effects of cold-pressed and distilled pomelo essential oils. Ascorbic acid and D-limonene were used as positive and negative controls, respectively. Droplines indicate the $50 \%$ clearance of DPPH and the corresponding dosage, ED50, of each sample. essential oils and their major volatiles showed similar results in which approximately $85 \%$ of DPPH was retained after reaction with D-limonene for $30 \mathrm{~min}^{38}$ Ascorbic acid, CPEO and DEO of Liangping pomelo exhibited significant scavenging effects in a dose dependent manner. Their ED50 values, as indicated with drop lines, were 0.28 $\mu \mathrm{mol}, 0.46 \mathrm{~mL}$ and $1.07 \mathrm{~mL}$ per $\mu \mathrm{mol}$ of DPPH. The antiradical power (ARP) of the essential oils was calculated and shown as the equivalent amount of ascorbic acid (Table 2). CPEO was $230 \%$ more powerful than DEO in scavenging DPPH free radicals.
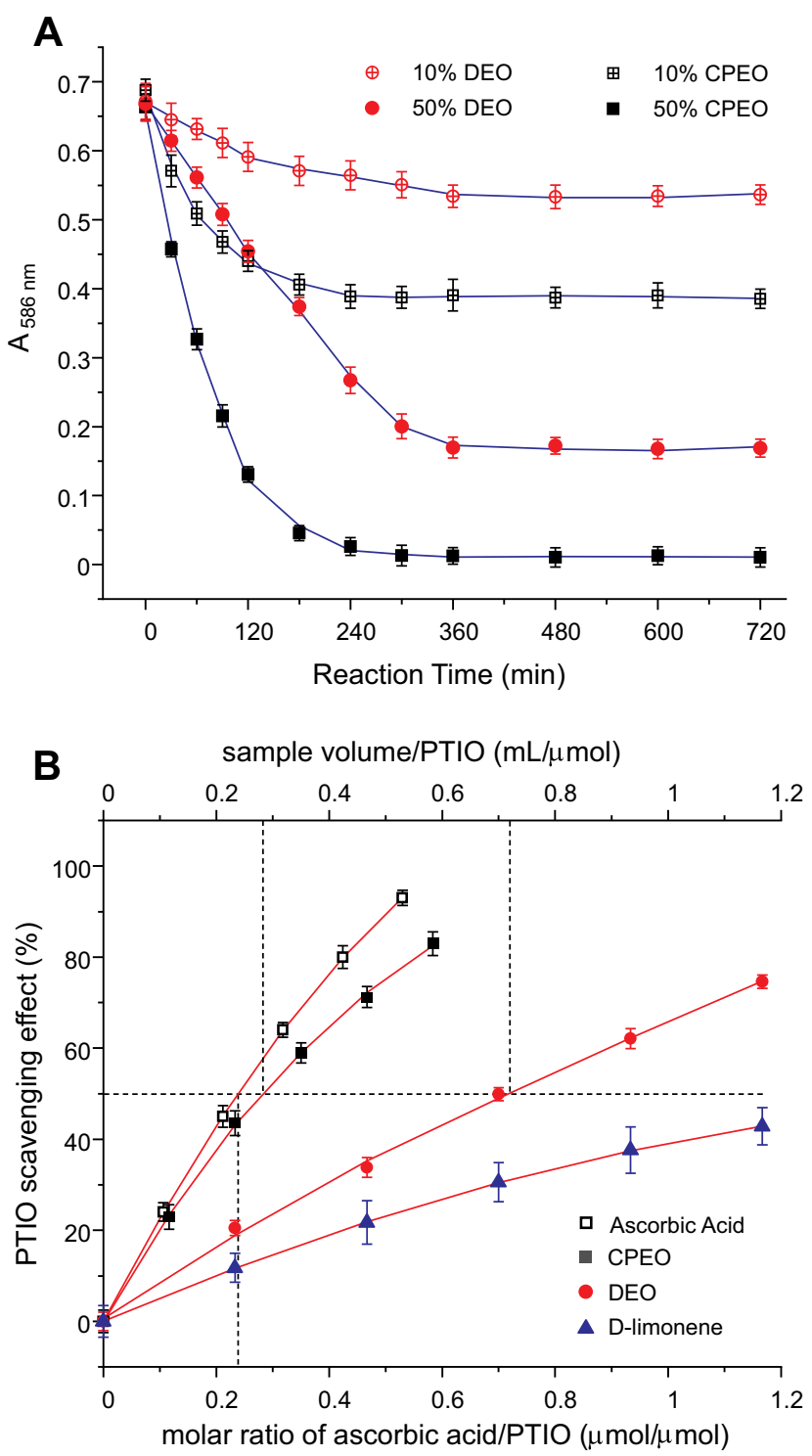

Figure 4 PTIO radical scavenging effects of pomelo essential oils. (A) Evolution of absorbance when reacted with $10 \%$ and $50 \%$ dilutions of CPEO or DEO; (B) scavenging rate of ascorbic acid, CPEO, DEO and D-limonene. Droplines indicate the $50 \%$ clearance of PTIO and the corresponding ED50 of each sample. 
PTIO is a stable ROS and can be directly determined using spectrophotometry. ${ }^{30}$ To find the best reaction time of essential oils with PTIO, we determined the absorbance decay of the mixture at $586 \mathrm{~nm}$ as a function of time. The evolution of $\mathrm{A}_{586 \mathrm{~nm}}$ within $12 \mathrm{~h}$ when reacted with low $(10 \%, v / v)$ and high $(50 \%, v / v)$ levels of CPEO and DEO is shown in Figure 4A. The fact that all absorbance curves were hyperbolic-like, reaching a stable minimum at approximately $4-6 \mathrm{~h}$, indicated that scavenging of PTIO by essential oils followed a slow kinetic pattern. A six-hours reaction was chosen, and the results of the effect-dose relations are shown in Figure 4B. All the samples showed higher scavenging effects on PTIO than on DPPH. The ED50 values of ascorbic acid, CPEO and DEO were $0.24 \mu \mathrm{mol}, 0.28 \mathrm{~mL}$ and $0.72 \mathrm{~mL}$, respectively, per $\mu \mathrm{mol}$ of PTIO. ARP value of CPEO was 2.6 times higher than that of DEO (Table 2).

\section{OHCs Structures and Bioactivity}

The results of FRAP, DPPH and PTIO assays showed the same order of antioxidant capacity, $\mathrm{CPEO}>\mathrm{DEO}>\mathrm{D}$-limonene. The multiple higher effects of cold pressed oil beyond distilled oil should be mainly attributed to the nonvolatiles. As shown in Figure 1, p-coumaric acid, ferulic acid and naringin, the most common phytochemical antioxidants were only detected in trace amounts in CPEO. They were more hydrophilic and hardly soluble in the terpene matrices of essential oil due to the multiple hydroxyl groups. Therefore, $\mathrm{OHCs}$ were the most likely contributor to the additional antioxidant activity. Similarly, Naseri et $\mathrm{al}^{39}$ reported that six furanocoumarins and coumarins extracted from Apiaceae roots were moderate DPPH scavengers.

Active hydrogens such as phenolic hydroxy- and mercapto- $\mathrm{H}$ are considered to mediate fast scavenging reactions. However, compounds with these functional groups were only detected at trace concentrations. The slow antiradical behavior of CPEO is mainly based on other structures in the OHCs. We propose that the most abundant epoxy moiety is a probable scavenger based on previous reports. De Almeida et $\mathrm{al}^{40}$ reported the quenching abilities of (+)-limonene epoxide against hydroxyl radical and thiobarbituric acid reactive species. They proposed a sequential mechanism comprising the epoxy-ring opening and the formation of a multimembered-radical transient state. Gurak et $\mathrm{al}^{41}$ found that $\beta$-carotene epoxides had a higher ability to scavenge ABTS radicals and singlet oxygen than (all-E)- $\beta$-carotene. In addition, comparatively active hydrogens such as the vicinal diol in the side chain of meranzin hydrate and $6^{\prime}, 7^{\prime}$-DHB, and allyl alcohol in the side-chain of auraptenol, could offer extra scavenging ability. Stobiecka ${ }^{42}$ studied the scavenging mechanism of ABTS and DPPH by geraniol, a volatile compound in citrus essential oils. They found that the presence of an allylic hydrogen atom close to the hydroxyl group was essential in the reaction. Nevertheless, they were probable minor contributors due to their low concentrations in CPEO.

In recent decades, due to the ever-growing occurrence of resistant strains of pathogens, limitations and abuse problems of antibiotics and chemical drugs have been profoundly recognized. The use of plant essential oils as complementary and alternative therapies against pathogenic microbes has attracted increasing attention. ${ }^{43,44}$ Donadu et $\mathrm{al}^{45-47}$ reported that essential oils from Hornstedtia bella, lavender and North Sardinia plants showed inhibitive activity on methicillin-resistant Staphylococcus aureus, multidrug-resistant Pseudomonas aeruginosa and Candida spp. Coumarins have been indicated as possible sources for the discovery and development of antimicrobial agents, and their activities are related to the substitution structures. For example, the hydroxyl-substituted congeners exhibit better antifungal activity against human pathogenic fungi than those lacking hydroxylation; substitution at the C-8 position of 7-hydroxycoumarins has been reported to have strong inhibitory activity. ${ }^{48,49}$ The cold-pressed essential oils of pomelo, which contain a high concentration and various types of bioactive coumarins and furanocoumarins, are promising natural antimicrobial agents affecting antibiotic/drugresistant pathogens.

\section{Conclusion}

The long unnoticed nonvolatiles of pomelo essential oil were comprehensively analyzed for their composition profile and antioxidant potential. OHCs, which are mainly composed of coumarins and furanocoumarins, were found to be the major constituents of the nonvolatiles. The cold-pressed essential oil of pomelo showed a significant in vitro antioxidant potency and a longacting radical scavenging activity. OHCs were the major active compounds contributing to the antioxidant effects. The results obtained will provide preliminary information for the further development of pomelo essential oils for the treatment of oxidation-related diseases. 


\section{Acknowledgments}

This research was funded by Natural Science Foundation of Chongqing, grant number cstc2019jcyj-bshX0104; Fundamental Research Funds for the Central Universities, grant number XDJK2020C026; Openproject of National Citrus Engineering Research Centre of China, grant number NCERC2019004; and Universitylocal Authorities Cooperation Project of Southwest University, grant number 2020001040. The authors are grateful to Yinchu Li and Suping Chen for their help regarding sample preparation. The authors also appreciate $\mathrm{Xi}$ Chen for her preliminary work on antioxidant study of various citrus essential oils.

\section{Disclosure}

Dr Leng Han reports grants from Science and Technology Bureau of Chongqing, grants from Ministry of Education of China, grants from National Citrus Engineering Research Centre of China, during the conduct of the study. The authors report no other conflicts of interest in this work.

\section{References}

1. Sun H, Ni H, Yang Y, Chen F, Cai H, Xiao A. Sensory evaluation and gas chromatography-mass spectrometry (GC-MS) analysis of the volatile extracts of pummelo (Citrus maxima) peel. Flav Frag J. 2014;29 (5):305-312. doi:10.1002/ffj.3206

2. Ou MC, Liu YH, Sun YW, Chan CF. The composition, antioxidant and antibacterial activities of cold-pressed and distilled essential oils of Citrus paradisi and Citrus grandis (L.) osbeck. Evid Based Complement Alternat Med. 2015;2015:804091. doi:10.1155/2015/ 804091

3. Settanni L, Palazzolo E, Guarrasi V, et al. Inhibition of foodborne pathogen bacteria by essential oils extracted from citrus fruits cultivated in Sicily. Food Control. 2012;26(2):326-330. doi:10.1016/j. foodcont.2012.01.050

4. Singh P, Shukla R, Prakash B, et al. Chemical profile, antifungal, antiaflatoxigenic and antioxidant activity of Citrus maxima burm. and Citrus sinensis (L.) osbeck essential oils and their cyclic monoterpene, DL-limonene. Food Chem Toxicol. 2010;48(6):1734-1740. doi:10.1016/j.fct.2010.04.001

5. He WY, Li XY, Peng Y, He XY, Pan SY. Anti-oxidant and anti-melanogenic properties of essential oil from peel of pomelo cv. Guan Xi. Molecules. 2019;24(2):13.

6. Hariya $T$. The possibility of regulating the function of adipose cells by odorants. Aroma Res. 2003;13:72-78.

7. Dugo G, Cotroneo A, Bonaccorsi I, Restuccia M. The composition of the volatile fraction of peel oils. In: Dugo G, Bonaccorsi I, editors. Citrus Bergamia - Bergamot and Its Derivatives. Boca Raton, FL: CRC Press; 2019:115-225.

8. Haro-Guzmán L. Composition of distilled oils. In: Giovanni dugo LM, editor. Citrus Oils: Composition, Advanced Analytical Techniques, Contaminants, and Biological Activity. Vol 49. 1st ed. Boca Raton, FL: Taylor \& Francis Group; 2011:193-217.

9. Njoroge SM, Koaze H, Karanja PN, Sawamura M. Volatile constituents of redblush grapefruit (Citrus paradisi) and pummelo (Citrus grandis) peel essential oils from Kenya. J Agric Food Chem. 2005;53(25):9790-9794. doi:10.1021/jf051373s
10. Minh tu NT, Thanh LX, Une A, Ukeda H, Sawamura M. Volatile constituents of Vietnamese pummelo, orange, tangerine and lime peel oils. Flav Frag J. 2002;17(3):169-174. doi:10.1002/ffj.1076

11. Sawamura M, Shichiri K-I, Ootani Y, Zheng XH. Volatile constituents of several varieties of pummelos and characteristics among citrus species. Agric Biol Chem. 1991;55(10):2571-2578.

12. Goh RMV, Pua A, Liu SQ, et al. Characterisation of volatile and non-volatile compounds in pomelo by gas chromatography-olfactometry, gas chromatography and liquid chromatography-quadrupole time-of-flight mass spectrometry. $J$ Essent Oil Res. 2020;32(2):132-143. doi:10.1080/ 10412905.2019.1677272

13. Mondello L, Dugo P, Cavazza A, Dugo G. Characterization of essential oil of pummelo (cv. Chandler) by GC/MS, HPLC and physicochemical indices. J Essent Oil Res. 1996;8(3):311-314. doi:10.1080/ 10412905.1996.9700622

14. Dugo P, Russo M. The oxygen heterocyclic components of citrus essential oils. In: Giovanni dugo LM, editor. Citrus Oils: Composition, Advanced Analytical Techniques, Contaminants, and Biological Activity. Vol 49. New York: CRC Press; 2010:405-444.

15. Fayek NM, Farag MA, Abdel Monem AR, Moussa MY, AbdElwahab SM, El-Tanbouly ND. Comparative metabolite profiling of four citrus peel cultivars via ultra-performance liquid chromatography coupled with quadrupole-time-of-flight-mass spectrometry and multivariate data analyses. J Chromatogr Sci. 2019;57(4):349-360. doi:10.1093/chromsci/bmz006

16. Li G, Tan F, Zhang Q, et al. Protective effects of polymethoxyflavone-rich cold-pressed orange peel oil against ultraviolet B-induced photoaging on mouse skin. $J$ Funct Foods. 2020;67:103834. doi:10.1016/j.jff.2020.103834

17. Valko M, Leibfritz D, Moncol J, Cronin MTD, Mazur M, Telser J. Free radicals and antioxidants in normal physiological functions and human disease. Int J Biochem Cell Biol. 2007;39(1):44-84.

18. Mutlu-Ingok A, Devecioglu D, Dikmetas DN, Karbancioglu-Guler F, Capanoglu E. Antibacterial, antifungal, antimycotoxigenic, and antioxidant activities of essential oils: an updated review. Molecules. 2020;25(20):4711. doi:10.3390/molecules 25204711

19. Amorati R, Foti MC, Valgimigli L. Antioxidant Activity of Essential Oils. J Agric Food Chem. 2013;61(46):10835-10847. doi:10.1021/ jf403496k

20. Diniz Do Nascimento L, Barbosa de Moraes AA, Santana da Costa K, et al. Bioactive natural compounds and antioxidant activity of essential oils from spice plants: new findings and potential applications. Biomolecules. 2020;10(7):988. doi:10.3390/biom10070988

21. Nguyen Thi Lan P, Pham Van H, Pham Thi Lan C, Nguyen Hoang D. Impact of extraction methods on antioxidant and antimicrobial activities of citrus essential oils. J Essent Oil Bear Pl. 2015;18 (4):806-817. doi:10.1080/0972060X.2014.977565

22. Wu Z, Li H, Luo Y, et al. Insights into the structural characterisations, bioactivities and their correlations with water-soluble polysaccharides extracted from different pomelo (Citrus maxima Merr.) tissues. Int $J$ Food Sci Technol. 2020;55(9):3091-3103. doi:10.1111/ ijfs. 14573

23. Li G-J, Wang J, Cheng Y-J, et al. Prophylactic effects of polymethoxyflavone-rich orange peel oil on $N^{\omega}$-Nitro- $L$-Arginine-induced hypertensive rats. Appl Sci. 2018;8(5):752. doi:10.3390/app8050752

24. Chen Y, Wu J, Xu Y, Fu M, Xiao G. Effect of second cooling on the chemical components of essential oils from orange peel (Citrus sinensis). J Agric Food Chem. 2014;62(35):8786-8790. doi:10.1021/jf501079r

25. Li G-J, Wu H-J, Wang Y, Hung W-L, Rouseff RL. Determination of citrus juice coumarins, furanocoumarins and methoxylated flavones using solid phase extraction and HPLC with photodiode array and fluorescence detection. Food Chem. 2019;271:29-38. doi:10.1016/j. foodchem.2018.07.130 
26. Abraham J. Proceedings of the international conference on harmonization $(\mathrm{ICH})$ of technical requirements for registration of pharmaceuticals or human use. In: Tietje C, Brouder A, editors. Handbook of Transnational Economic Governance Regimes. Leiden: Martinus Nijhoff Publishers; 1996:1041-1053.

27. Benzie IFF, Strain JJ. Ferric reducing/antioxidant power assay: direct measure of total antioxidant activity of biological fluids and modified version for simultaneous measurement of total antioxidant power and ascorbic acid concentration. In: Packer L, editor. Methods in Enzymology. Vol 299. San Diego: Academic Press; 1999: 15-27.

28. Brand-Williams W, Cuvelier ME, Berset C. Use of a free radical method to evaluate antioxidant activity. LWT-Food Sci Technol. 1995;28(1):25-30. doi:10.1016/S0023-6438(95)80008-5

29. Du Z-X, Zhang Y, Sun Y, Gong S-Z. DPPH free radical scavenging and antimicrobial activity of the essential oil of pomelo peel. Flav Fragr Cosmet. 2017;(01):39-41+45.

30. Li X. 2-Phenyl-4,4,5,5-tetramethylimidazoline-1-oxyl 3-Oxide (PTIO•) radical scavenging: a new and simple antioxidant assay in vitro. J Agric Food Chem. 2017;65(30):6288-6297. doi:10.1021/ acs.jafc. 7 b02247

31. Bocco A, Cuvelier ME, Richard H, Berset C. Antioxidant activity and phenolic composition of citrus peel and seed extracts. J Agric Food Chem. 1998;46(6):2123-2129. doi:10.1021/jf9709562

32. Zhang MX, Duan CQ, Zang YY, Huang ZW, Liu GJ. The flavonoid composition of flavedo and juice from the pummelo cultivar (Citrus grandis (L.) Osbeck) and the grapefruit cultivar (Citrus paradisi) from China. Food Chem. 2011;129(4):1530-1536. doi:10.1016/j. foodchem.2011.05.136

33. Dugo P, Russo M. The oxygen heterocyclic components of citrus essential oils. In: Dugo G, Mondello L, editors. Citrus Oils: Composition, Advanced Analytical Techniques, Contaminants, and Biological Activity. Vol 49. 1st ed. Boca Raton, FL: Taylor \& Francis Group; 2011:193-217.

34. Christensen LP. Chapter 29 - Polyphenols and polyphenol-derived compounds from plants and contact dermatitis. In: Watson RR, Preedy VR, Zibadi S, editors. Polyphenols: Prevention and Treatment of Human Disease. 2nd ed. Academic Press; 2018:349-384.

35. Messer A, Raquet N, Lohr C, Schrenk D. Major furocoumarins in grapefruit juice II: phototoxicity, photogenotoxicity, and inhibitory potency vs. cytochrome P450 3A4 activity. Food Chem Toxicol. 2012;50(3-4):756-760. doi:10.1016/j.fct.2011.11.023

36. Naganuma M, Hirose S, Nakayama Y, Nakajima K, Someya T. A study of the phototoxicity of lemon oil. Arch Dermatol Res. 1985;278(1):31-36. doi:10.1007/BF00412492

37. Sun W, Rice MS, Park MK, et al. Intake of furocoumarins and risk of skin cancer in 2 prospective US cohort studies. J Nutr. 2020;150 (6):1535-1544. doi:10.1093/jn/nxaa062
38. Choi H-S, Song HS, Ukeda H, Sawamura M. Radical-scavenging activities of citrus essential oils and their components: detection using 1,1-diphenyl-2-picrylhydrazyl. J Agric Food Chem. 2000;48 (9):4156-4161. doi:10.1021/jf000227d

39. Naseri M, Monsef-Esfehani HR, Saeidnia S, Dastan D, Gohari AR. Antioxidative coumarins from the roots of Ferulago subvelutina. Asian J Chem. 2013;25(4):1875-1878. doi:10.14233/ ajchem.2013.13208

40. de Almeida AAC, de Carvalho RBF, Silva OA, de Sousa DP, de Freitas RM. Potential antioxidant and anxiolytic effects of (+)-limonene epoxide in mice after marble-burying test. Pharmacol Biochem Behav. 2014;118:69-78. doi:10.1016/j.pbb.2014.01.006

41. Gurak PD, Mercadante AZ, González-Miret ML, Heredia FJ, Meléndez-Martínez AJ. Changes in antioxidant capacity and colour associated with the formation of $\beta$-carotene epoxides and oxidative cleavage derivatives. Food Chem. 2014;147:160-169. doi:10.1016/j. foodchem.2013.09.106

42. Stobiecka A. Comparative study on the free radical scavenging mechanism exerted by geraniol and geranylacetone using the combined experimental and theoretical approach. Flav Fragr J. 2015;30 (5):399-409. doi:10.1002/ffj.3256

43. Le NT, Donadu MG, Ho DV, et al. Biological activities of essential oil extracted from leaves of Atalantia sessiflora Guillauminin Vietnam. J Infect Dev Ctries. 2020;14(9):1054-1064. doi:10.3855/ jidc. 12469

44. Barac A, Donadu M, Usai D, et al. Correction to: antifungal activity of Myrtus communis against Malassezia sp. isolated from the skin of patients with pityriasis versicolor. Infection. 2018;46(2):287. doi:10.1007/s15010-017-1104-2

45. Donadu MG, Trong Le N, Viet ho D, et al. Phytochemical compositions and biological activities of essential oils from the leaves, rhizomes and whole plant of Hornstedtia bella Škorničk. Antibiotics. 2020;9(6):334. doi:10.3390/antibiotics9060334

46. Donadu M, Usai D, Pinna A, et al. In vitro activity of hybrid lavender essential oils against multidrug resistant strains of Pseudomonas aeruginosa. J Infect Dev Ctries. 2018;12(1):9-14. doi:10.3855/ jidc. 9920

47. Donadu MG, Usai D, Marchetti M, et al. Antifungal activity of oils macerates of North Sardinia plants against candida species isolated from clinical patients with candidiasis. Nat Prod Res. 2020;34 (22):3280-3284. doi:10.1080/14786419.2018.1557175

48. Sardari S, Nishibe S, Daneshtalab M. Coumarins, the bioactive structures with antifungal property. Stud Nat Prod Chem. 2000;23:335-393.

49. Kayser O, Kolodziej H. Antibacterial activity of simple coumarins: structural requirements for biological activity. Z Naturforsch C. 1999;54(3-4):169-174. doi:10.1515/znc-1999-3-405

\section{Publish your work in this journal}

Drug Design, Development and Therapy is an international, peerreviewed open-access journal that spans the spectrum of drug design and development through to clinical applications. Clinical outcomes, patient safety, and programs for the development and effective, safe, and sustained use of medicines are a feature of the journal, which has also been accepted for indexing on PubMed Central. The manuscript management system is completely online and includes a very quick and fair peer-review system, which is all easy to use. Visit http://www. dovepress.com/testimonials.php to read real quotes from published authors. 